\title{
Improving road safety in small towns based on a systematic approach
}

\author{
Irina Makarova ${ }^{1}$, Gulnara Yakupova $^{1 *}$, and Eduard Mukhametdinov ${ }^{1}$ \\ ${ }^{1}$ Kazan Federal University, Syuyumbike Avenue, 10A, Naberezhnye Chelny, 423812, Russia
}

\begin{abstract}
Currently improving road safety is the priority task in the field of transport management. In our opinion, making management decisions to reduce the road accidents number and injuries rate should be based on a systematic approach to identifying the accidents' causes and severity. This can be achieved using, among other things, the analysis of statistical data on violations and accidents in dynamics. A model so-called the Haddon Matrix was used as a method of preliminary identification of influencing factors and possible measures for their elimination. The statistical analysis results are presented a particular settlement. It was revealed that the city specifics, namely the residents' number, motorization rate and the road network and infrastructure characteristics, are reflected in the factors affecting the level of accidents rate and, as a consequence, on the measures necessary to reduce it.
\end{abstract}

\section{Introduction}

The acceleration of urbanization causes the growth of megacities and, as a result, the need for mobility and motorization of their population. Along with population motorization growth, the number and size of commercial vehicle parks is increasing. Given an insufficiently developed infrastructure, this leads to an increase in the number of road accidents (RA), as well as the severity of their consequences. All this causes a decrease in the transport system stability and safety as a whole.

Events related to COVID-19 have changed the parameters of the transport system's functioning. Due to local bans on travel by private automobile transport, the level of drivers' professional skill, determined, among other things, by the regularity of driving, has decreased. After the ban on movement for both pedestrians and drivers was lifted, the risk of accidents increased significantly.

To create an effective traffic control system, it is necessary to have a tool with which it would be possible to identify the most significant factors from the actual data, to play various scenarios of the events' development, to make corrective decisions, and subsequently, to analyze their effectiveness.

* Corresponding author: math8@mail.ru 


\section{Methology of the system approach in the road safety managment}

The effectiveness of a management strategy based on a systems approach is confirmed by numerous examples of researchers from many European countries [1, 2, 3, 4], who argue that through a combination of technical and organizational measures, it is possible to predict and prevent most road accidents, as well as reduce the severity consequences.

Due to the intellectualization and digitalization of all processes and systems, the intelligent transport system (ITS) is one of the main tools of improving road safety. The introduction of ITS is associated with the use of modern communication facilities, the development of data processing algorithms, monitoring and control methods, using onboard computers, sensors and other technical means, which are equipped with modern vehicles and infrastructure facilities [5,6]. Effective management decisions are formed on the basis of information received in real time [7,8].

The researchers in [9] decline that the vehicles' intellectualization will increase the stability of transport systems. The authors show the methods of situational management based on intelligent technologies to improve the safety of complex technical systems. They developed an object-oriented case model that describes the dynamics of dangerous conditions object. Measures are provided for identifying dangerous conditions that entail the occurrence of accidents, identifying their causes, assessing safety, predicting development scenarios.

To improve traffic safety, various measures are also proposed, consisting in optimizing the design of unsafe road sections [10], introducing systems for monitoring traffic violations [11] and driver fatigue [12], using real-time systems to predict road safety indicators [13] and decision support systems based on data mining [14].

Thus, given the effectiveness of the systematic approach noted in scientific and government views on safety issues, for identifying typical influencing factors and measures for their decreasing the road policy should include the development of effective management actions in three directions: to prevent accidents, to reduce their severity and to eliminate the accident consequences.

Accident injuries are reduced by measures to improve passive safety: seat belts and sensors, triplex windows, child restraints, protective helmets, an elastic impact absorbing element for pedestrian's protection, fences with a structure that ensures the gradual "consumption" of impact energy, artificial road bumps.

Accident injuries are reduced by measures to improve passive safety: seat belts and sensors, triplex windows, child restraints, protective helmets, an elastic impact absorbing element for pedestrian's protection, fences with a structure that ensures the gradual "consumption" of impact energy, artificial road bumps.

To eliminate the accident's consequences, the efficiency and speed of the emergency services are important; to alert them, emergency call systems in vehicles, as well as computer vision technologies that recognize an accident that has occurred are provided.

\section{Results: identification of typical situations leading to reduced road safety In Yelabuga town}

Solving the safety problems of transport systems is a priority area outlined in the "Road Safety Strategy in the Russian Federation for 2018-2024" [15].

The specifics of traffic control depend on both the planning solution and the city's size. For example, the Yelabuga city, located in the Tatarstan Republic, was taken. It has a 
population of 73,913 people, which, according to the classification, gives reason to attribute it to medium-sized cities, but having a high level of motorization.

The specifics of the traffic organization in Yelabuga is one-way traffic, which contributes to increasing throughput, and also eliminates the conflict of oncoming traffic flows with insufficient width of the carriageway.

An analysis of the initial information by accident rate received by the Road Safety State Inspectorate in the Yelabuga city for 2017-2019 in terms of accident rate showed that the number of accidents is growing annually, and, as a result, the number of victims and deaths is growing (Figure 1a). The severity of the consequences, calculated as the share of those died in accidents out of the total number of victims (dead and wounded), for 2019 year amounted to $8.7 \%$. At the same time, in almost $100 \%$ of cases there was a violation of traffic rules by traffic accidents participants, in $9 \%$ of cases the vehicle driver was intoxicated, in $23 \%$ of cases unsatisfactory conditions of detention and arrangement of the road network were recorded.

Figure 1b, showing the violations' dynamics over the past three years, shows an annual increase (by about 20\%) in violations that increase the accidents' probability, while the level of violations that increase the severity of theit consequences remains stable. This dynamics is due to the growing number of automatic photo-video recording of violations, as well as the expansion of their capabilities: the ability to notice violations of several types at once.

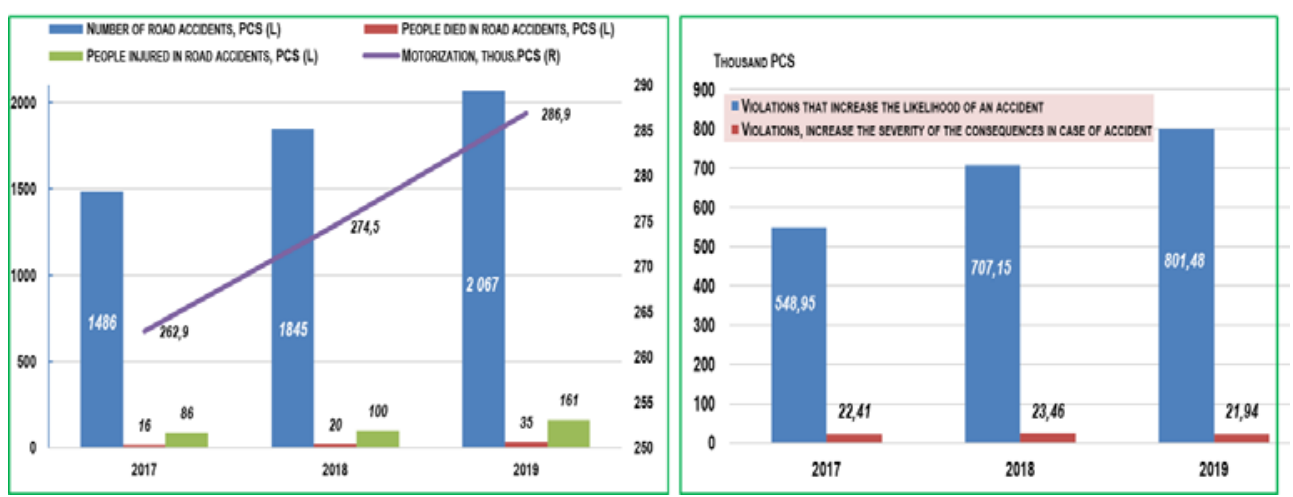

Fig. 1. a) Dynamics of motorization and accident rates in Yelabuga for 2017-2019, b) Dynamics of violations in Yelabuga for 2017-2019

To determine the risk of accidents in various types of traffic violations, the data on the revealed facts of traffic violations and accidents were compared (Figure 2). In recent years, the decrease in the number of the most common type of violations (speeding, or noncompliance with the chosen speed with specific road conditions) is primarily due to the active introduction of photo and video recording cameras. At the same time, a significant number of accidents are associated with a violation of the rules for the vehicles' location on the roadway. This situation is explained by the peculiarities of the city's infrastructure, characterized by a small territory, where with the growth of motorization there is a shortage of parking spaces, both for the vehicles' permanent storage (10342 places), and for its temporary stop (5474 places). 


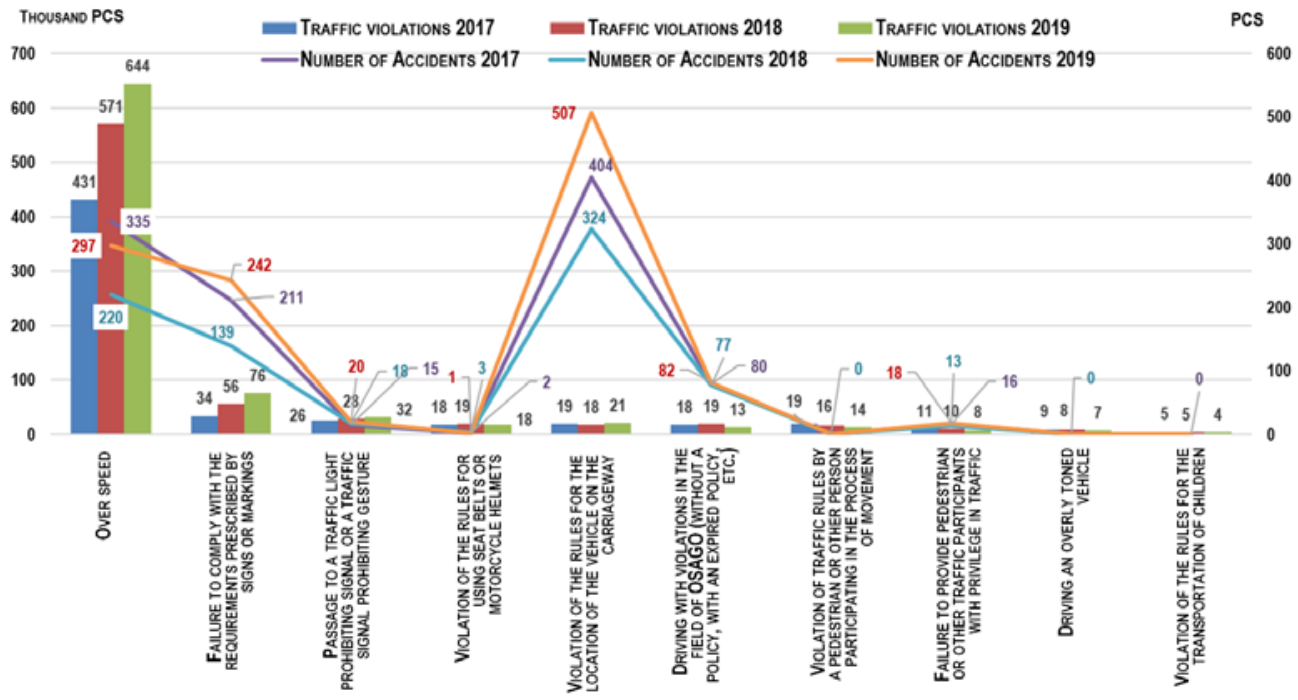

Fig. 2 Relationship of traffic violations and the number of accidents in Yelabuga for 2017-2019

The maximum load is observed at the intersection of Tugarov and Moskovskaya streets, which is $58 \%$ of the throughput. There are other lively unregulated and adjustable intersections. The absence of traffic lights or suboptimal parameters of their operation lead to the accumulation of a large number of vehicles that cause congestion at such intersections, thereby increasing the accident's likelihood. In this regard, there is a need to optimize traffic and the quality of setting the parameters of traffic lights operation. One can test the effectiveness of possible solutions to this problem using simulation of specific situations.

It is also worth noting that the budget for small and medium cities is very limited, therefore, it is not advisable to begin large-scale reconstruction of the street and road infrastructure, which requires large material costs, without pre-development activities and play it on the simulation model.

The analysis of the places of concentration of road accidents revealed the need to introduce regulation at the intersection of Neftyanikov Avenue with Okruzhnoye and Tanaevskoye Highways in Yelabuga to reduce the likelihood and severity of accidents.

The solution may be to install a traffic light at this intersection, which, with adequate adjustment of its operating parameters, can streamline traffic, reduce accidents by separating pedestrian and traffic flows, eliminate ambiguity in the interpretation of situations by drivers and ensure safe crossing of the intersection by pedestrians and cyclists.

Additionally, when organizing an adjustable pedestrian crossing and arranging pedestrian railings, an unregulated pedestrian crossing near house 12 on Neftyanikov Avenue can be transferred to this intersection, which will also increase safety in this area in terms of traffic management.

You can check the effectiveness of this solution using simulation. The AnyLogic ${ }^{\circ}$ software package, which has a built-in traffic library and OptionQuest optimizer, was chosen as an environment for building a simulation model. The structure of the model is shown in Fig. 3a.

When optimizing the operating mode of a traffic light object, transport delays at the intersection and the average length of the congestion at the approaches to the intersection are analyzed. 

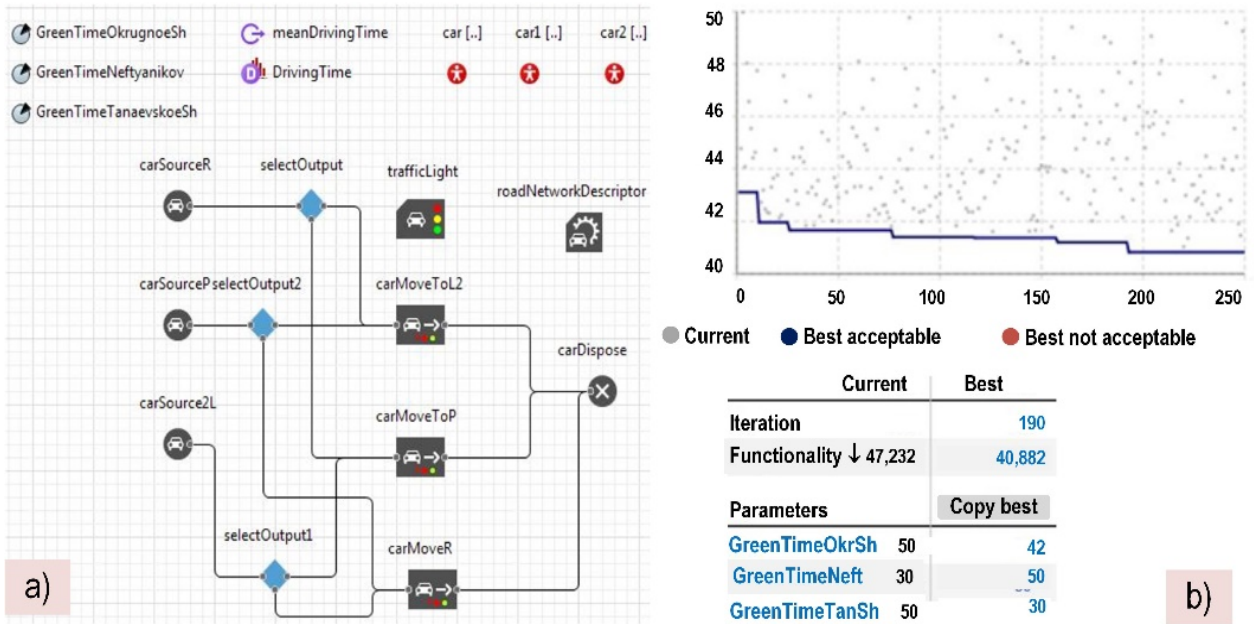

Fig. 3 a) the structure of the crossroad simulation model; b) results of the optimization experiment

We determined the logic of the traffic light: a two-phase cycle, in which the first phase is a green light for driving along the Okruzhnoye Highway in all permitted directions, and the second is a green light for entering the intersection from Neftyanikov Avenue and from the Tanaevskoye Highway. When determining the optimal parameters, it was assumed that the infrastructure changes would be minimal and would consist in optimizing the operation of the installed traffic light object. Therefore, the objective function is the average travel time of the road section of the car, and the influencing parameters are the cycle times of the green and red traffic light signals, by changing which the traffic light operation is optimized. The optimal values obtained during the simulation experiment are shown in Fig. $3 \mathrm{~b}$. In the process of optimization, the best value of the functional was obtained, at which congestion on the considered section of the road traffic system would be reduced, the probability of road accidents and negative impacts on the environment would decrease (Fig. 4 ), and the average time of crossing this intersection in each direction would decrease.

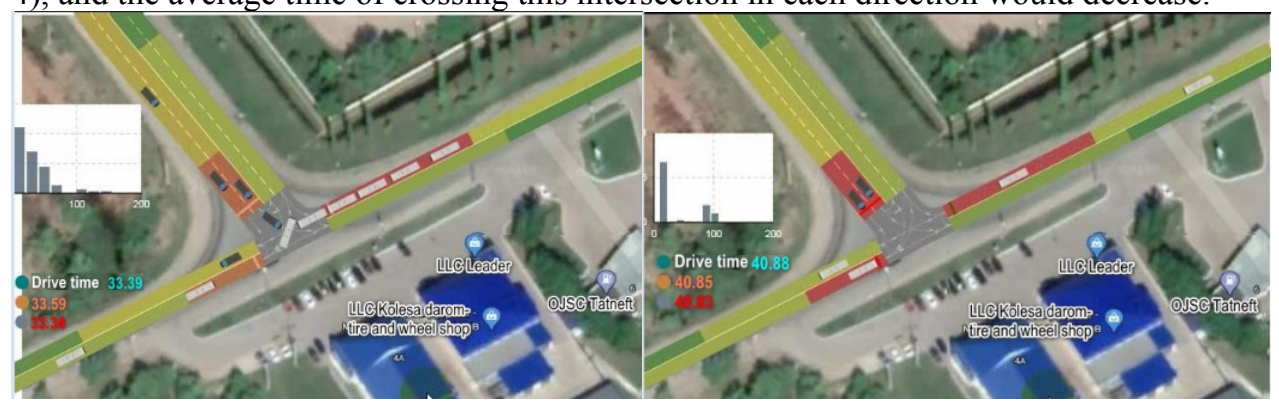

Fig. 4 Type of the IM of the UDS section of the city of Elabuga: a) before the changes; b) after the changes

The found phase durations can be used when introducing elements of an intelligent traffic planning system, including adaptive traffic lights. Their use is an affordable solution that does not require a large-scale reconstruction of the engineering structures of the transport infrastructure, associated with high material costs.

The problems identified by the analysis require specific measures to improve road safety, which can be consolidated into a Haddon matrix. In particular, at this stage, transport routes' and infrastructure parameters' optimization is appropriate for the initial implementation (Figure 5). This methodology, [16], is an example of a systematic approach 
that allows to identify risk factors and develop events to implement a planned and effective management system to prevent and reduce the harmful effects of road traffic injuries. In the studies of William Haddon, factors are subdivided into human, automobile, environmental factors. They are considered in the context of three time phases - before the accident, during the accident and after the accident. In our opinion, due to the fact that the processes of intellectualization affected both vehicles and infrastructure, a new factor has appeared in the traffic control system, which can be indicated by IT or artificial intelligence (AI). So, we modified the Haddon matrix. In any case, after the implementation of the events, regathering of statistics and analysis is necessary to understand the effectiveness of the decisions taken. You may then need to adjust the events in the Haddon matrix.

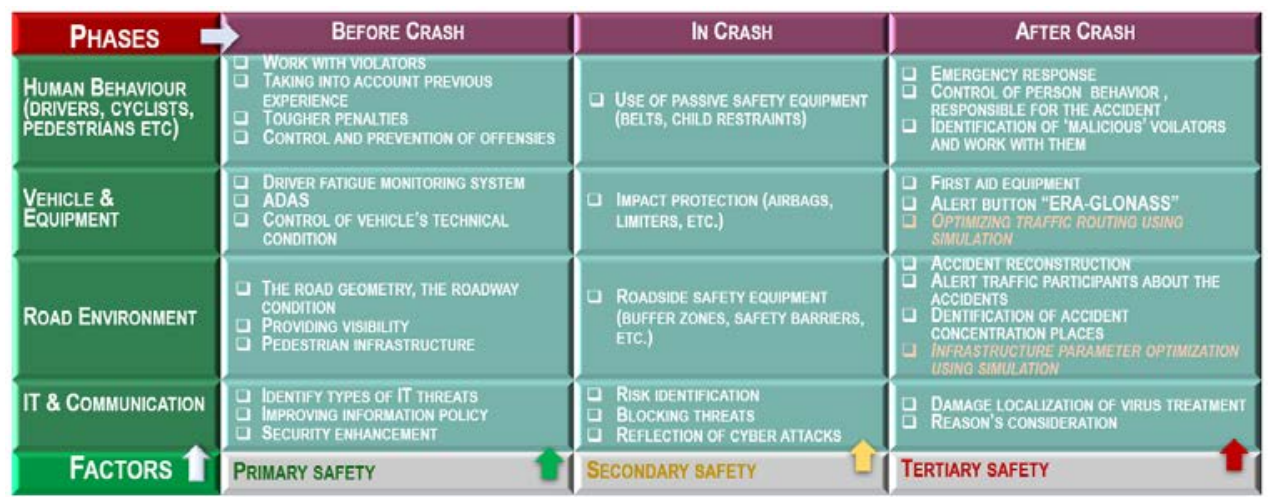

Fig. 5 Modified Haddon matrix

\section{Conclusions}

To reduce the likelihood of accidents in small and medium-sized cities, according to the analysis, measures aimed at improving management and improving road infrastructure may be implemented. Since most of the accidents are caused by the incorrect vehicles' location on the roadway, it can be concluded that the installation of photo-video recording cameras did not have a significant effect. Alternative low-cost events to expand the parking space should be recommended.

Since the budgets of small and medium-sized cities are small, as an effective budgetary tool, first, it is necessary to provide for management measures to optimize the parameters of already existing infrastructure facilities and to record them in the Haddon matrix, for example, adjusting the phases of traffic lights. At the same time, simulation modeling is an effective tool for assessing the adequacy of the decisions made.

As an example, the Elabuga city was considered, the influencing factors were found, and the problematic section of the road network was identified. The option of installing a traffic light at a selected intersection is considered. Two series of experiments were carried out, during which the number of traffic light phases and their duration were varied, the optimal duration of the operating modes was found, which ensured the minimum travel time of the section by vehicles.

It should be noted that the prerequisites for the Haddon matrix successful application are the collection and systematization of information about ongoing activities, and, subsequently, about the parameters of infrastructure and the environment, traffic monitoring data, as well as information about road users and accidents. Only in this case the feedback principle is realized and it becomes possible to assess the Haddon Matrix effectiveness in the transport system management. 


\section{Acknowledgements}

The reported study was funded by RFBR, project number 19-29-06008 $\backslash 20$.

\section{References}

1. Zero mortality: how does the Swedish concept of Vision Zero work?

http://www.aif.ru/auto/safety/obnulit_smertnost_kak_rabotaet_shvedskaya_koncepciya _vision_zero.

2. R.S. Jurecki. Analysis of Road Safety in Poland after Accession to the European Union, Communications. (vol. 22, no. 2, pp. 60-67, 2020).

3. R. Buehler, J. Pucher. The growing gap in pedestrian and cyclist fatality rates between the United States and the United Kingdom, Germany, Denmark, and the Netherlands, 1990-2018. Transport Reviews. Advance online publication. doi:10.1080/01441647.2020.1823521 (2021).

4. ITF (2020), "Monitoring Progress in Urban Road Safety", International Transport Forum Policy Papers, No. 79, OECD Publishing, Paris.

5. V. Shepelev, S. Aliukov, K. Nikolskaya, S. Shabiev. The capacity of the road network: Data collection and statistical analysis of traffic characteristics. Energies. Volume 13, Issue 7, p. 1765 (2020)

6. K. Khazukov, V. Shepelev, T. Karpeta, S. Shabiev, I. Slobodin, I. Charbadze, I. Alferova. Real-time monitoring of traffic parameters. Journal of Big Data, Volume 7, Issue 1, p. 84 (2020)

7. Makarova, R. Khabibullin, E. Belyaev, V. Mavrin. Increase of city transport system management efficiency with application of modeling methods and data intellectual analysis. Studies in Systems, Decision and Control. Volume 32, pp. 37-80 (2016).

8. V. Shepelev, S. Aliukov, A. Glushkov, S. Shabiev. Identification of distinguishing characteristics of intersections based on statistical analysis and data from video cameras. Journal of Big Data, Volume 7, Issue 1, p. 46 (2020)

9. Makarova, A. Pashkevich, E. Mukhametdinov, V. Mavrin. Application of the situational management methods to ensure safety in intelligent transport systems. VEHITS 2017 - Proceedings of the 3rd International Conference on Vehicle Technology and Intelligent Transport Systems 2017, Pages 339-345 (2017)

10. H. Yao Zhao and D. Long. Evaluation of Highway traffic safety based on velocity dispersion. Proceedings 2011 International Conference on Transportation, Mechanical, and Electrical Engineering (TMEE), pp. 141-145 (2011)

11. M. Divyaprabha, M. Thangavel and P. Varalakshmi. A Comparative Study on Road Safety Problems. 2018 IEEE International Conference on Computational Intelligence and Computing Research (ICCIC), pp. 1-7 (2018)

12. Beying Deng and Xufeng Zhang. Car networking application in vehicle safety. 2014 IEEE Workshop on Advanced Research and Technology in Industry Applications (WARTIA), pp. 834-837 (2014)

13. Z. Peng, S. Gao, Z. Li, B. Xiao and Y. Qian. Vehicle Safety Improvement through Deep Learning and Mobile Sensing. IEEE Network, vol. 32, no. 4, pp. 28-33 (2018)

14. H. El Alaoui El Abdallaoui, A. El Fazziki, F. Z. Ennaji and M. Sadgal. Decision Support System for the Analysis of Traffic Accident Big Data. 14th International 
Conference on Signal-Image Technology \& Internet-Based Systems (SITIS), Las Palmas de Gran Canaria, pp. 514-521 (2018)

15. Federal target program of the Russian Federation "Improving road safety in 20132020", approved by the order of the Government of the Russian Federation, No. 864 (2013)

16. Jr. W. Haddon. Options for the prevention of motor vehicle crash injury. Israel Journal of Medical Sciences, Volume 16, Issue 1, pp. 45-65, (1980) 